\title{
Real-world prevalence and incidence of lower back pain in Germany
}

\author{
Karel Kostev ${ }^{1}$ (D)
}

Received: 11 March 2019 / Accepted: 21 March 2019 / Published online: 27 March 2019

(c) Springer-Verlag GmbH Germany, part of Springer Nature 2019

Fatoye et al. performed a systematic review, and reported the incidence and prevalence rates of lower back pain based on studies using routinely collected data. The review contains data from seven countries, including Canada, the United States of America, Sweden, Belgium, Finland, Israel, and The Netherlands [1]. In their study, the prevalence of lower back pain ranged from 1.4 to $20.0 \%$, with incidences from 0.02 to $7.0 \%$.

The authors stated that further research studies are required to estimate prevalence and incidence, and identify the risk factors for lower back pain using patients' electronic medical records.

As no data from Germany were included in the review, I performed an analysis of the prevalence and incidence of lower back pain in Germany. My analysis used data from the Disease Analyzer database (IQVIA), which compiles drug prescriptions, diagnoses, and basic medical and demographic data obtained directly and anonymously from computer systems used in the practices of general practitioners and specialists [2].

The database contained a total of $2,367,885$ patients with at least one visit to one of 1000 general practitioners' offices in Germany between January 2017 and December 2017. Of these patients, $46.7 \%$ were male and $28.0 \%$ were $\geq 65$ years of age.

Prevalence was defined as the proportion of patients with at least one documentation of lower back pain (ICD-10: M54.5) in 2017. Incidence was calculated as the proportion of patients with a first-time documentation of lower back pain in 2017.

Lower back pain was diagnosed in 88,660 patients (prevalence $=3.7 \%$ ). Lower back pain was documented in a significantly higher share of men than women (4.1\% versus $3.4 \%)$,

Karel Kostev

kkostev@de.imshealth.com

1 Epidemiology, IQVIA, Unterschweinstiege 2-14, 60549 Frankfurt am Main, Germany but no significant difference was found between people aged $<65$ versus $\geq 65$ years $(3.7 \%$ versus $3.8 \%)$.

In 30,817 patients (incidence $=1.3 \%$ ), lower back pain was diagnosed for the first time. No significant differences were found between women and men (1.3\% in both groups) or people aged $<65$ versus $\geq 65$ years ( $1.3 \%$ in both groups).

Our results are in line with the results reported by Fatoye et al., but further research studies are needed in other countries.

\section{Compliance with ethical standards}

Conflict of interest Karel Kostev declares that he has no conflicts of interest related to this article.

Research involving human participants/informed consent German law allows the use of anonymous electronic medical records for research purposes under certain conditions. According to this legislation, it is not necessary to obtain informed consent from patients or approval from a medical ethics committee for this type of observational study that contains no directly identifiable data. Since patients were only queried as aggregates and no protected health information was available for queries, no IRB approval was required for the use of this database or the completion of this study.

\section{References}

1. Fatoye F, Gebrye T, Odeyemi I (2019) Real-world incidence and prevalence of low back pain using routinely collected data. Rheumatol Int. https://doi.org/10.1007/s00296-019-04273-0

2. Rathmann W, Bongaerts B, Carius HJ, Kruppert S, Kostev K (2018) Basic characteristics and representativeness of the German Disease Analyzer database. Int J Clin Pharmacol Ther 56(10):459-466

Publisher's Note Springer Nature remains neutral with regard to jurisdictional claims in published maps and institutional affiliations. 